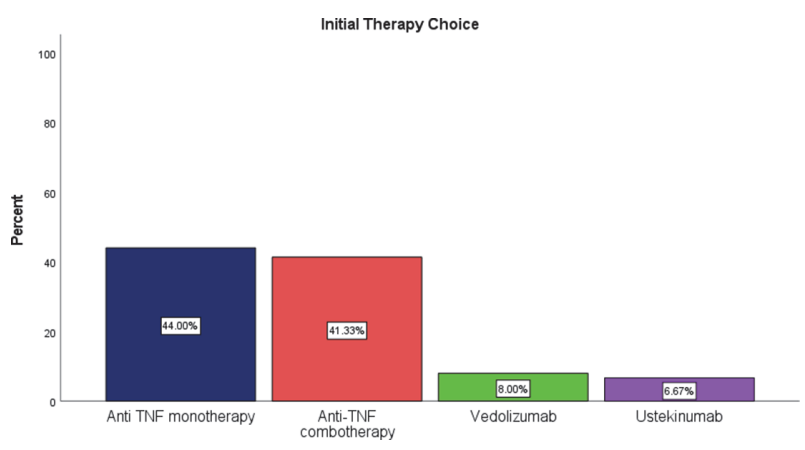

Abstract PM0-41 Figure 1 Initial Theraphy choice

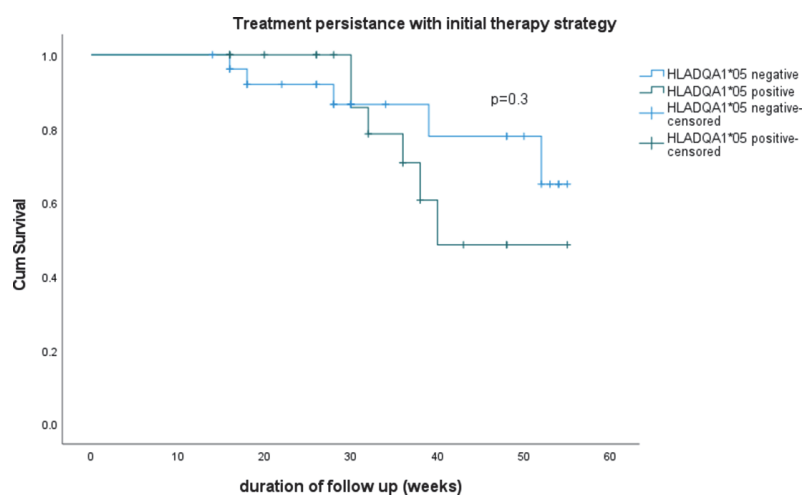

Abstract PM0-41 Figure 2 Treatment presistance with initial theraphy strategy

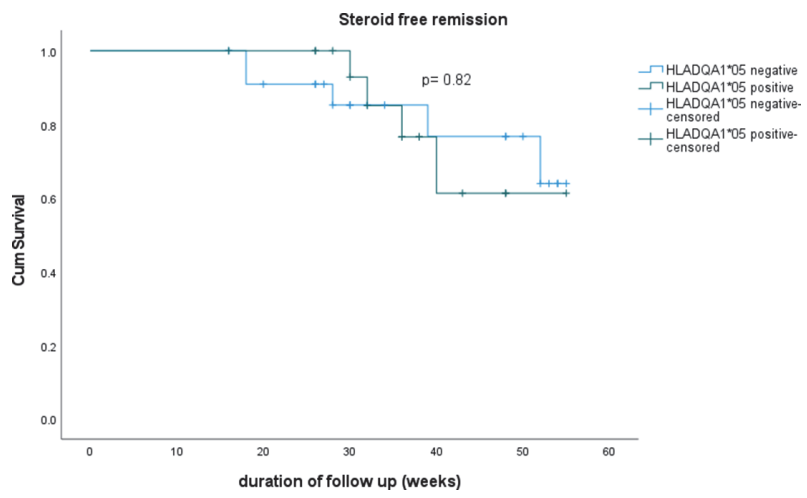

Abstract PM0-41 Figure 3 Steroid free remission

with 7 out of 10 positive for HLADQA $1 * 05$. However, only $3(4.6 \%)$ had undetectable drug levels in the presence of antibody and all three were HLADQA1*05 positive. Two patients had reactions during induction therapy both were HLADQA1*05 positive and were on combination therapy with Infliximab. Therapy persistence with initial drug strategy and steroid free remission at 6 months was recorded in $77.1 \%$ and $78 \%$ respectively. There was no significant difference in drug persistence rates at 6 months and 12 months in patients with HLADQA1*05 variant or those with variant absent (Figure 2). Steroid free remission at 6 and 12 months was also similar irrespective of the variant status (Figure 3)

Conclusions Choice of therapy incorporating HLADQA1*05 status may allow anti-TNF monotherapy and tailoring of therapy in IBD patients. A randomised stratified biomarker trial is required to determine the utility of pre-treatment testing.
PM0-42 ACCELERATED DOSING SCHEDULE WITH USTEKINUMAB IN ANTI-TNF REFRACTORY CROHN'S DISEASE

Haidee Aleman Gonzalez*, Katie Stamp, Emma Whitehead, Sally Myers, Jack Turnbull, Alison Pattinson, Alison Talbot, Shaji Sebastian. Hull University Teaching Hospitals Nhs Trust, Hull, UK

\subsection{6/gutjnl-2021-BSG.181}

Introduction The efficacy of ustekinumab in prior biologic exposed refractory patients with inflammatory bowel disease is significantly inferior to those who are bio naïve. The optimal therapeutic level and hence the optimal dosing strategy in this setting is uncertain. Whether early dose optimisation is beneficial in this group of patients has not been evaluated. We aimed to compare the effectiveness and safety of early dose optimisation for maintenance compared to conventional maintenance regime in a retrospective cohort of refractory IBD patients.

Methods All patients initiated on ustekinumab following failure of one or more anti-TNF agents were included. Patients who received conventional maintenance regime (Q8W) was compared to those who received accelerated maintenance regime $(\mathrm{Q} 4 \mathrm{H})$. The primary end point was steroid free remission at 12 months or at last follow up. The secondary end points were ustekinumab persistence at 6 months and 12 months, need for surgery and drug related infectious or non-infectious complications.

Results One hundred and four patients were included (male: female 48:56). Median follow up was 11 months (IQR 5-14). Fifty-six patients received accelerated dosing regimen while 48 patients received standard dosing regime. Dose escalation was required in 18 patients $(37.5 \%)$ of standard dosing regimen while four patients $(7.1 \%)$ in the accelerated dosing regimen had dose de-escalation. Higher proportion patients in the accelerated dosing regimen were in steroid free remission $87.5 \%$ vs $68.8 \%(p=0.018)$. Proportion of patients requiring surgery was higher in the standard dosing regimen group (22.91 5 vs $7.14 \%, p=0.02)$. Ustekinumab persistence in patient started on the accelerated regime and conventional dosing regime was not significantly different at 6 months $(94.1 \%$ and $95.3 \%, p=0.58)$ and 12 months $(84.4 \%$ and 95.3, $\mathrm{p}=0.1)$. No serious drug related complications observed in either group.

Conclusions Early dose optimisation of Ustekinumab improves steroid free remission rates and reduces the need for surgery in patients with anti-TNF refractory patients with IBD.

\section{PMO-43 COLIFORM-SENSITIVITY BASED ORAL ERTAPENEM THERAPY FOR CHRONIC ANTIBIOTIC-REFRACTORY POUCHITIS: AN UNDERUTILISED OPTION?}

Thomas Conley*, Ibrahim Mian, Kate Martin, Paul Rooney, Sreedhar Subramanian. Liverpool University Foundation Trust, Liverpool, UK

\subsection{6/gutjnl-2021-BSG.182}

Introduction Up to $50 \%$ of patients have at least one episode of pouchitis after ileal pouch anal-anastomosis (IPAA). Among these, a proportion develop chronic antibiotic-dependent or chronic antibiotic refractory pouchitis (CARP). Pouch failure is common among patients with CARP. Faecal coliform sensitivity testing in such patients can identify a subgroup in whom an alternative, more appropriate antimicrobial agent can be utilised. We previously reported successful coliform sensitivity- 